\section{BRAZIULIAN JOURNAL \\ OF MEDICAL AND BIOLOGICAL RESHARCH}

www.bjournal.com.br
ISSN 0100-879X

Volume 43 (12) 1135-1244 December 2010

BIOMEDICAL SCIENCES

AND

CLINICAL INVESTIGATION

Braz J Med Biol Res, December 2010, Volume 43(12) 1153-1159

doi: 10.1590/S0100-879X2010007500127

Rabbit model of uncontrolled hemorrhagic shock and hypotensive resuscitation

J.B. Rezende-Neto, S.B. Rizoli, M.V. Andrade, T.A. Lisboa and J.R. Cunha-Melo

The Brazilian Journal of Medical and Biological Research is partially financed by

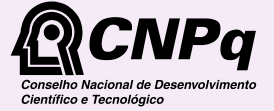

Ministério Cientifico e Tecrológico

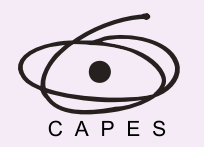

Ministério da Educação

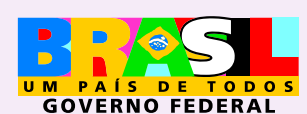

GOVERNO FEDERAL
DTAPESP

Institutional Sponsors
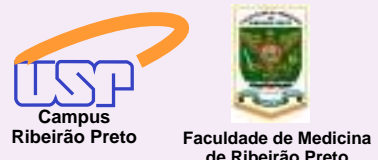

Ф SHIMADZU

GE Healthcare
Hotsite of proteomics metabolomics developped by: 


\title{
Rabbit model of uncontrolled hemorrhagic shock and hypotensive resuscitation
}

\author{
J.B. Rezende-Neto ${ }^{1,3}$, S.B. Rizoli' ${ }^{4}$, M.V. Andrade ${ }^{2}$, \\ T.A. Lisboa ${ }^{1}$ and J.R. Cunha-Melo ${ }^{1}$ \\ 1Departamento de Cirurgia, ${ }^{2}$ Departamento de Clínica Médica, Faculdade de Medicina, \\ ${ }^{3}$ Hospital Universitário Risoleta Tolentino Neves, \\ Universidade Federal de Minas Gerais, Belo Horizonte, MG, Brasil \\ ${ }^{4}$ Sunnybrook Health Sciences Centre, University of Toronto, Ontario, Canada
}

\begin{abstract}
Clinically relevant animal models capable of simulating traumatic hemorrhagic shock are needed. We developed a hemorrhagic shock model with male New Zealand rabbits (2200-2800 g, 60-70 days old) that simulates the pre-hospital and acute care of a penetrating trauma victim in an urban scenario using current resuscitation strategies. A laparotomy was performed to reproduce tissue trauma and an aortic injury was created using a standardized single puncture to the left side of the infrarenal aorta to induce hemorrhagic shock similar to a penetrating mechanism. A 15-min interval was used to simulate the arrival of pre-hospital care. Fluid resuscitation was then applied using two regimens: normotensive resuscitation to achieve baseline mean arterial blood pressure (MAP, 10 animals) and hypotensive resuscitation at $60 \%$ of baseline MAP (10 animals). Another 10 animals were sham operated. The total time of the experiment was $85 \mathrm{~min}$, reproducing scene, transport and emergency room times. Intraabdominal blood loss was significantly greater in animals that underwent normotensive resuscitation compared to hypotensive resuscitation ( $17.1 \pm 2.0$ vs $8.0 \pm 1.5 \mathrm{~mL} / \mathrm{kg}$ ). Antithrombin levels decreased significantly in normotensive resuscitated animals compared to baseline (102 \pm 2.0 vs $59 \pm 4.1 \%$ ), sham ( $95 \pm 2.8$ vs $59 \pm 4.1 \%$ ), and hypotensive resuscitated animals $(98 \pm 7.8$ vs $59 \pm 4.1 \%$ ). Evidence of re-bleeding was also noted in the normotensive resuscitation group. A hypotensive resuscitation regimen resulted in decreased blood loss in a clinically relevant small animal model capable of reproducing hemorrhagic shock caused by a penetrating mechanism.
\end{abstract}

Key words: Hemorrhagic shock; Animal model; Resuscitation

\section{Introduction}

Severe hemorrhage accounts for approximately $40 \%$ of all trauma-related deaths (1). Hemorrhage is the leading cause of preventable death and the first following admission to a hospital, particularly among patients in need of urgent surgery, with the death of $80 \%$ of those who die during an operation being due to hemorrhage (2). Therefore, reducing early mortality caused by hemorrhage is a major objective of research both at the experimental and clinical levels (3-5).

Animal models have played a crucial role in hemorrhagic shock research. Almost all advances and interventions in hemorrhage have been initially or concomitantly described in such models. They have become more sophisticated and capable of addressing hemostatic and physiological responses to hemorrhage (6-10). However, a major challenge faced by all experimental models is to fully mimic the human condition and being able to address many aspects of traumatic hemor- rhagic shock in a clinically relevant manner (6-10)

There are basically three major types of experimental models for hemorrhagic shock: fixed volume hemorrhage, fixed pressure hemorrhage and uncontrolled hemorrhage. Some models use a combination of strategies even though they provoke distinct hemodynamic and hemostatic responses $(11,12)$. Most experts agree that an ideal and clinically relevant model of traumatic hemorrhagic shock should incorporate severe uncontrolled hemorrhage and tissue injury. In addition, the model's fluid resuscitation strategy should be in keeping with current clinical practice where either normotensive resuscitation - for rapid restoration of end-organ perfusion - or the more recent approach of hypotensive resuscitation for penetrating torso injuries could be applied $(3,13)$. Another important aspect of a model of traumatic hemorrhage is to be consistent, as closely as

Correspondence: J.B. Rezende-Neto, Rua Dr. Riggi, 133, 34000-000 Nova Lima, MG, Brasil. Fax: +55-31-3581-7126.

E-mail: jbrezende.ufmg@gmail.com

Received July 2, 2010. Accepted October 29, 2010. Available online November 19, 2010. Published December $20,2010$. 
possible, with the transport times and fluid infusion rates of emergency medical systems $(14,15)$.

The purpose of the present study was to develop a clinically relevant rabbit model of uncontrolled hemorrhagic shock that simulates penetrating torso trauma. The model also involved normotensive and hypotensive resuscitation regimens.

\section{Material and Methods}

The study was approved by the Animal Research Committee of Universidade Federal de Minas Gerais (CETEA/ UFMG, Protocol \#203/2007), Belo Horizonte, MG, Brazil. Animal experiments were performed according to a protocol approved by the University Ethics Committee in Animal Experimentation. Male New Zealand rabbits (2200 to 2800 g), $60-70$ days old, were maintained at $25^{\circ} \mathrm{C}$ on a $12-\mathrm{h}$ light/dark cycle. The animals were housed individually in an authorized facility of the Faculty of Medicine (UFMG) and were given rabbit chow (Nutricoelho, Purina, Brazil) and water ad libitum. All animals were acclimated for 2 weeks before the experiment.

\section{Animal preparation}

Animals were anesthetized with $60 \mathrm{mg} / \mathrm{kg}$ ketamine and $8 \mathrm{mg} / \mathrm{kg}$ xylazine (Fort Dodge Animal Health, USA) administered intramuscularly. Additional doses were administered intravenously as needed (15 mg/kg ketamine and $2 \mathrm{mg} /$ $\mathrm{kg}$ xylazine). The operative sites were prepared with $10 \%$ povidone iodine solution. A tracheotomy was performed and a 3-cm segment of a polyethylene catheter $(4.7 \mathrm{~mm}$ in inner diameter) was partially inserted into the trachea. The right internal jugular vein and the right carotid artery were cannulated with polyethylene tubing (PE10; Clay Adams, USA) previously filled with lactated Ringer's (LR) solution. A 5-cm midline laparotomy was performed under sterile conditions, and 2-0 nylon continuous full thickness running sutures were placed through the edges of the laparotomy for later abdominal closure.

\section{Baseline blood samples}

Immediately after the laparotomy phase, blood samples (a total of $3 \mathrm{~mL}$ ) were obtained from the carotid artery for the following baseline tests: arterial blood gas (Siemens, Germany) in a 1-mL sample in a heparinized syringe, complete blood count and platelet count (STKS Coulter, USA) in a 1-mL sample in a vacuum blood collection tube containing ethylenediaminetetraacetic acid (Vacuette, Greiner Bio-one, Brazil), and activated partial thromboplastin time (aPTT) (Sysmex CA-540, USA) and antithrombin activity (STAGO, USA) in a 1-mL sample in a vacuum blood collection tube containing $3.8 \%$ sodium citrate.

\section{Experimental groups \\ Thirty animals were randomly divided into three groups}

of 10 animals each. Sham group animals underwent the same preparation and baseline blood sampling as the other two groups, but not hemorrhagic shock. The normotensive resuscitation (NR) group received an intravenous LR solution to maintain mean arterial pressure (MAP) at baseline (pre-shock) levels. The hypotensive resuscitation (HR) group received LR to maintain MAP at $60 \%$ of baseline levels.

\section{Hemorrhagic shock procedure}

All animals were placed on a heating pad, which was not heated until intravenous fluid infusion was started and baseline rectal temperature was assessed. The right carotid artery tubing was connected to a Pro-Paq (Protocol Systems, USA) for continuous MAP monitoring. Ten minutes were allowed for stabilization of the parameters before baseline MAP was obtained. During this time the abdomen was covered with a plastic film to reduce insensible fluid losses. Hemorrhagic shock was then induced by a standardized single-puncture injury to the left side of the infrarenal aorta, $2 \mathrm{~mm}$ below the lower pole of the left kidney, with a 16-gauge needle (16 G Jelco ${ }^{\circledR}$ Medex, USA). The abdomen was immediately closed by pulling the previously placed sutures. For the next 15 min no fluids were infused to simulate the average time emergency medical services (EMS) would take to arrive at the scene (Figure 1). Fifteen minutes after the beginning of shock, LR solution at $39^{\circ} \mathrm{C}$ was infused continuously through the right jugular vein catheter with an infusion pump (Stoelting Co., USA). The infusion rate was set to maintain MAP at the predetermined levels for each group, with a $5 \mathrm{~mL} / \mathrm{min}$ maximum rate. Also at this time the heating pad was turned on to maintain rectal temperature above $35^{\circ} \mathrm{C}$. Fluid resuscitation was continued until $85 \mathrm{~min}$ after the aortic injury to simulate average transport and emergency room times. The infusion rate was reduced every time goal MAP was achieved and total LR volume was recorded for each group. A new set of blood samples for the previously described laboratory tests were obtained after 85 min, i.e., at the end of the experiment. In addition, the abdomen was opened and total intra-abdominal blood loss was calculated as the difference between blood-soaked sponges minus the weight of pre-weighed dry sponges. Animals were killed at the end of the experiment with a ketamine and xylazine overdose and oblique section of the abdominal aorta.

\section{Statistical analysis}

Data are reported as means \pm SEM. Statistical analysis consisted of one-way analysis of variance (ANOVA) with post hoc analysis using the Tukey test for multiple comparisons between experimental means. The unpaired $t$-test was used to analyze comparisons between two groups. The level of significance was set at $\mathrm{P}<0.05$ for all analyses.

\section{Results}

\section{Mortality}

The overall mortality of animals subjected to hemor- 
rhagic shock was $20 \%$ (5 of 25 animals). All 5 deaths occurred before the beginning of fluid resuscitation. Three deaths occurred within 5 min after injury to the aorta and 2 at approximately 14 min after the injury; all dead animals were replaced. A total of 20 animals survived the injury to the aorta and consequently the hemorrhagic shock. There were no deaths among sham-operated animals $(N=10)$.

\section{Physiological variables and intra-abdominal bleeding}

There were no statistically significant differences in baseline MAP between groups: $66.5 \pm 1.6$ and $65.1 \pm 1.3$ $\mathrm{mmHg}$ in the sham and NR groups, respectively, and $67.8 \pm$ 4.2 in the HR group. Five minutes after aortic injury MAP was similarly reduced in the NR group $(17.3 \pm 1.2 \mathrm{mmHg})$ and $\mathrm{HR}$ group (15.4 $\pm 1.4 \mathrm{mmHg} ; \mathrm{P}>0.05)$ to a significantly lower level in both groups compared to sham-operated animals $(63.7 \pm 2.0 \mathrm{mmHg} ; \mathrm{P}<0.05)$. The MAP of the NR group was restored to levels similar to those of sham-operated animals at 30 min after the beginning of shock (50.2 \pm 4.5 ; $P>0.05)$ with the infusion of LR solution. However, as expected, the MAP of the HR group remained at $60 \%$ of baseline, a significantly lower level than in the other two groups, throughout the resuscitation phase up to the 85th $\min (41.4 \pm 2.8 ; \mathrm{P}>0.05$; Figure 2).

Baseline rectal temperature varied between $37.5 \pm 0.2$ and $37.9 \pm 0.1(P>0.05)$. At the end of the experiment (85th $\mathrm{min}$ ), the rectal temperature of sham-operated animals was $36.9 \pm 0.1^{\circ} \mathrm{C}$ compared to $36.0 \pm 0.3^{\circ} \mathrm{C}$ for shocked animals $(P>0.05)$.

Animals that underwent normotensive resuscitation bled significantly more from the aortic injury than animals that underwent hypotensive resuscitation $(17.5 \pm 2.8$ vs $8.1 \pm$ $1.8 \mathrm{~mL} / \mathrm{kg} ; \mathrm{P}<0.05$; Figure 3).

\section{Fluid infusion}

Significantly more intravenous fluid was required by the NR group animals to maintain their MAP at baseline levels $(123.2 \pm 10.1 \mathrm{~mL} / \mathrm{kg}$ ) compared to sham-operated animals

Figure 3. Intra-abdominal blood loss assessed at the 85th min by weighing blood-soaked sponges $(1 \mathrm{~g}=1 \mathrm{~mL})$. Sham = no hemorrhage $(\mathrm{N}=10$ rabbits); NR = normotensive resuscitation to baseline values $(\mathrm{N}=10$ rabbits); $\mathrm{HR}=$ hypotensive resuscitation to $60 \%$ of baseline mean arterial pressure ( $N=10$ rabbits). Data are reported as means \pm SEM. ${ }^{*} \mathrm{P}<0.05$ compared to hypotensive resuscitation (one-way ANOVA followed by the post hoc Tukey test for multiple comparisons).

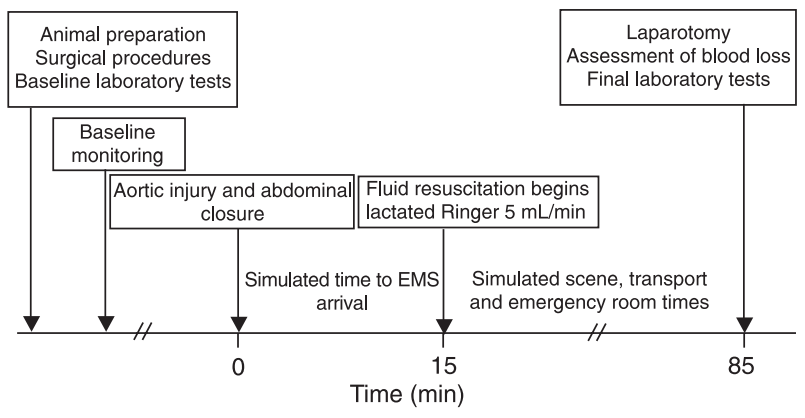

Figure 1. Timeline of the experiment. EMS = emergency medical services.

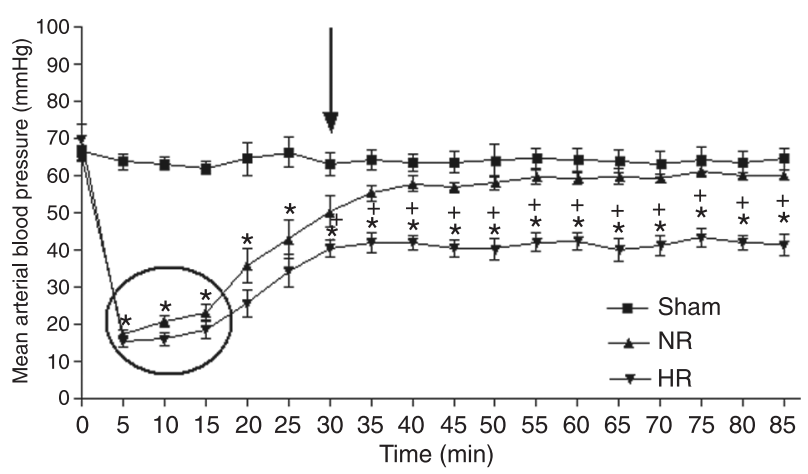

Figure 2. Effect of hemorrhage on mean arterial blood pressure of rabbits subjected to uncontrolled hemorrhage by aortic injury. The circle indicates the bleeding response and "self-resuscitation"; the arrow indicates the time when the normotensive resuscitation (NR) group ( $\mathrm{N}=10$ rabbits) achieved baseline mean arterial blood pressure; $\mathrm{HR}=$ hypotensive resuscitation group $(\mathrm{N}$ $=10$ rabbits) with mean arterial pressure maintained at $60 \%$ of baseline values; sham group ( $\mathrm{N}=10$ rabbits) subjected only to surgical procedures including laparotomy, but no hemorrhagic shock. Data are reported as means \pm SEM. ${ }^{*} \mathrm{P}<0.05$ compared to sham; ${ }^{+} \mathrm{P}<0.05$ compared to normotensive resuscitation (oneway ANOVA followed by the post hoc Tukey test for multiple comparisons).

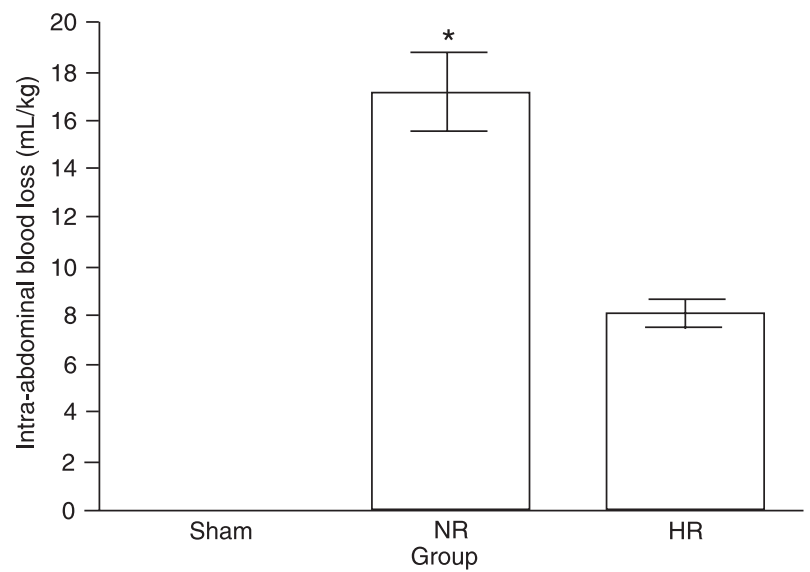


$(21.0 \pm 4.3 \mathrm{~mL} / \mathrm{kg})$ and to the HR group $(59 \pm 1.2 \mathrm{~mL} / \mathrm{kg}$; $\mathrm{P}<0.05$; Figure 4). Furthermore, over time, the NR group required significantly more fluid than the other two groups, as particularly noticed after the 50th min of the experiment, during the resuscitation phase (Figure 5).

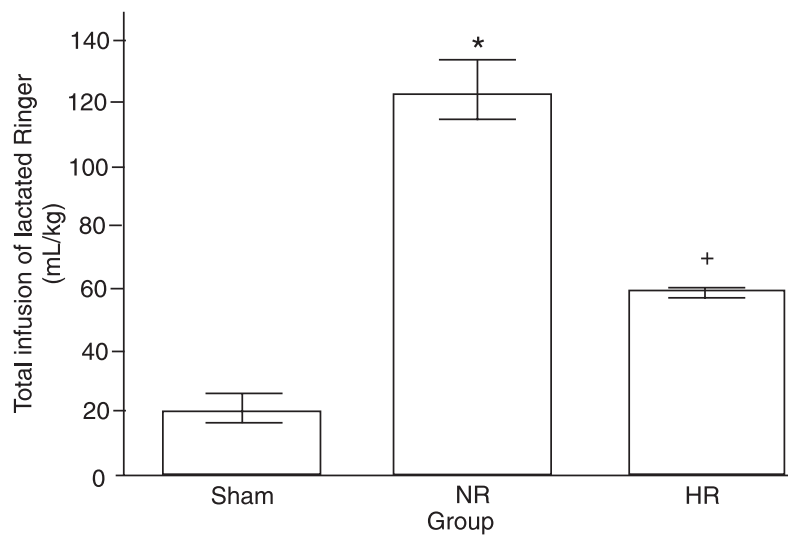

Figure 4. Total intravenous fluid infusion volume (lactated Ringer) infused through the right internal jugular vein catheter. Sham $=$ no hemorrhage $(\mathrm{N}=10$ rabbits $) ; \mathrm{NR}=$ normotensive resuscitation to baseline values ( $\mathrm{N}=10$ rabbits); $\mathrm{HR}=$ hypotensive resuscitation to $60 \%$ of baseline mean arterial pressure $(\mathrm{N}$ $=10$ rabbits). Data are reported as means \pm SEM. ${ }^{*} \mathrm{P}<0.05$ compared to sham and hypotensive resuscitation; ${ }^{+} \mathrm{P}<0.05$ compared to sham (one-way ANOVA followed by the post hoc Tukey test for multiple comparisons).

\section{Laboratory tests}

Baseline hemoglobin, hematocrit, and arterial blood gases were equivalent among groups (Table 1). As expected, because of hemorrhagic shock and resuscitation, hemoglobin decreased significantly in the NR $(6.9 \pm 2.0 \mathrm{~g} / \mathrm{dL})$ and $\mathrm{HR}(7.2 \pm 1.2 \mathrm{~g} / \mathrm{dL})$ groups compared to sham-operated

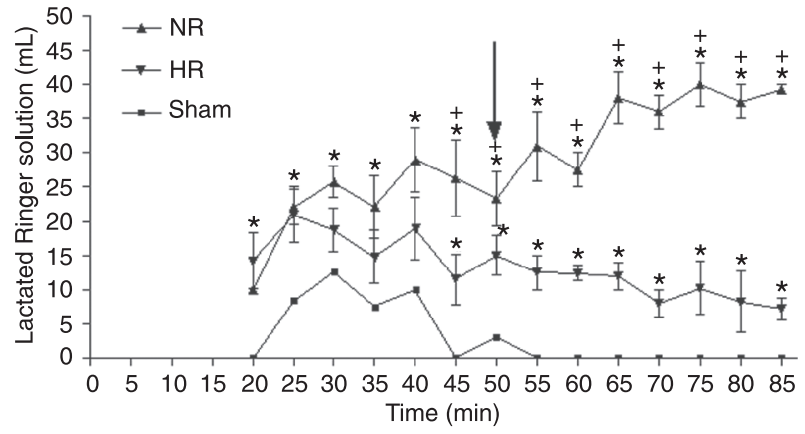

Figure 5. Fluid volume during time of lactated Ringer infusion through the right internal jugular vein catheter. The arrow indicates the time when re-bleeding possibly begins. Sham $=$ no hemorrhage $(\mathrm{N}=10$ rabbits $)$; $\mathrm{NR}=$ normotensive resuscitation to baseline values ( $\mathrm{N}=10$ rabbits); $\mathrm{HR}=$ hypotensive resuscitation to $60 \%$ of baseline mean arterial pressure $(\mathrm{N}=10$ rabbits). Data are reported as means \pm SEM. ${ }^{*} \mathrm{P}<0.05$ compared to sham; ${ }^{+} \mathrm{P}<0.05$ compared to sham and hypotensive resuscitation (one-way ANOVA followed by the post hoc Tukey test for multiple comparisons).

Table 1. Hemoglobin, hematocrit, arterial blood gases, platelets and antithrombin levels of sham and hemorrhaged animals submitted to different resuscitation strategies.

\begin{tabular}{|c|c|c|c|c|c|c|}
\hline \multirow[t]{2}{*}{ Test } & \multicolumn{2}{|c|}{ Sham } & \multicolumn{2}{|c|}{ NR } & \multicolumn{2}{|c|}{ HR } \\
\hline & Baseline & $85 \min$ & Baseline & $85 \min$ & Baseline & $85 \mathrm{~min}$ \\
\hline $\mathrm{Hg}(\mathrm{g} / \mathrm{dL})$ & $12.5 \pm 0.4$ & $12.2 \pm 0.2$ & $12.3 \pm 0.5$ & $6.9 \pm 2.0^{*}$ & $12.2 \pm 0.5$ & $7.2 \pm 1.2^{*}$ \\
\hline HCT $(\%)$ & $38.6 \pm 1.0$ & $36.9 \pm 0.9$ & $36.1 \pm 1.3$ & $23.0 \pm 2.5^{\star}$ & $36.2 \pm 0.8$ & $24.0 \pm 3.1^{*}$ \\
\hline $\mathrm{pH}$ & $7.4 \pm 0.6$ & $7.32 \pm 0.5$ & $7.41 \pm 0.2$ & $7.1 \pm 1.1$ & $7.4 \pm 0.3$ & $7.2 \pm 1.5$ \\
\hline $\mathrm{PaCO}_{2}(\mathrm{mmHg})$ & $50.0 \pm 2.3$ & $44.0 \pm 2.4$ & $49 \pm 1.8$ & $24.8 \pm 3.1^{*}$ & $51.1 \pm 1.2$ & $25.4 \pm 2.3^{*}$ \\
\hline $\mathrm{PaO}_{2}(\mathrm{mmHg})$ & $75.1 \pm 1.4$ & $83.0 \pm 3.4$ & $78.2 \pm 3.5$ & $158.0 \pm 22.1^{*}$ & $72.8 \pm 2.8$ & $160.1 \pm 16.0^{*}$ \\
\hline $\mathrm{BE}(\mathrm{mmol} / \mathrm{L})$ & $2.3 \pm 1.5$ & $0.7 \pm 1.1$ & $3.2 \pm 2.5$ & $-9.2 \pm 3.0^{*}$ & $4.0 \pm 1.3$ & $-9.8 \pm 3.2^{*}$ \\
\hline aPTT & $0.95 \pm 0.5$ & $0.9 \pm 0.3$ & $0.91 \pm 0.2$ & $1.1 \pm 0.5$ & $0.92 \pm 0.5$ & $0.94 \pm 0.2$ \\
\hline Platelets $\left(\mathrm{mm}^{3}\right)$ & $4.0 \times 10^{5} \pm 0.6$ & $3.8 \pm 10^{5} \pm 0.5$ & $3.7 \times 10^{5} \pm 0.4$ & $2.1 \times 10^{5} \pm 0.2^{*}$ & $3.2 \times 10^{5} \pm 0.4$ & $2.2 \times 10^{5} \pm 0.5^{*}$ \\
\hline Antithrombin (\%) & $99.8 \pm 2.0$ & $95 \pm 2.8$ & $102 \pm 2.0$ & $59.0 \pm 4.1^{*+}$ & $100.1 \pm 2.1$ & $98 \pm 7.8$ \\
\hline
\end{tabular}

$\mathrm{Hg}=$ hemoglobin; $\mathrm{HCT}=$ hematocrit, $\mathrm{PaCO}_{2}=$ partial carbon dioxide pressure in arterial blood; $\mathrm{PaO} 2=$ partial oxygen pressure in arterial blood; $\mathrm{BE}=$ base excess; aPTT = activated partial thromboplastin (time ratio vs control value); sham = no hemorrhage; NR = normotensive resuscitation group after hemorrhage; $\mathrm{HR}=$ hypotensive resuscitation group after hemorrhage. ${ }^{*} \mathrm{P}<0.05$ vs baseline of same group and sham group at baseline and $85 \mathrm{~min} ;{ }^{+} \mathrm{P}<0.05 \mathrm{vs} \mathrm{HR}$ at baseline and $85 \mathrm{~min}$ (one-way ANOVA followed by the post hoc Tukey test for multiple comparisons). 
animals $(12.2 \pm 0.2 \mathrm{~g} / \mathrm{dL}, \mathrm{P}<0.05)$. Similar results were obtained for hematocrit, even though HR animals received significantly less fluid (Table 1). Regarding arterial blood gases at the 85th min of the experiment, mean arterial $\mathrm{pH}$ was $7.1 \pm 1.1$ in the NR group and $7.2 \pm 1.5$ in the HR group. Data also showed evidence of compensatory hyperventilation with a significant decrease in $\mathrm{PaCO}_{2}$ levels among shocked animals (Table 1). As expected, base excess was significantly worse in the shock groups compared to baseline and to sham-operated animals (Table 1).

Baseline aPTT ratios, platelet count and antithrombin levels were similar among groups (Table 1). There were no significant differences in aPTT ratios after hemorrhagic shock. Platelet count decreased equivalently in both hemorrhagic shock groups compared to baseline and to shamoperated animals (Table 1). However, antithrombin level was only significantly reduced in the NR group compared to baseline, to the HR group and to sham-operated animals $(P<0.05)$.

\section{Discussion}

The current trauma literature has repeatedly called for better clinically relevant experimental models that more closely mimic hemorrhagic shock in humans $(6,9,10,15)$. We have devised a small animal model that reproduces many aspects of hemorrhage caused by a penetrating injury to the torso. The model also simulates fluid resuscitation prior to definitive surgical bleeding control. The lag time between vessel injury and the beginning of fluid resuscitation permits blood to clot at the site of injury. This is extremely important in terms of clinical relevance in any hemorrhagic shock model related to trauma $(15,16)$. Clot formation can be determined by whole blood clotting time and takes an average of 10 min to occur (16). Consequently, any experimental model in which fluid infusion begins before clot formation interferes with the physiological process of hemostasis and jeopardizes clinical relevance.

The present model also simulates the average time urban EMS take to arrive at the scene of an accident (approximately $15 \mathrm{~min}$ ), and this time is also allotted in our model before fluid resuscitation is started (14). Furthermore, we also consider a total time of $85 \mathrm{~min}$ for EMS transport and emergency room times from the time of injury. An audit of time to emergency trauma laparotomy in the United Kingdom showed a median time from emergency call to operation of $127 \mathrm{~min}$ (140 $\mathrm{min}$ for blunt and $86 \mathrm{~min}$ for penetrating injuries), which is similar to the time allotted in our model (17).

Most trauma patients in severe hemorrhagic shock also require blood product transfusion. However, the time to start the first transfusion varies considerably. A recent study showed that the beginning of transfusion of the first unit of red blood cells occurred on average 20 min after trauma, and the time to first fresh frozen plasma transfusion ranged from 24 to 350 min (median $93 \mathrm{~min}$ ) (18).

Overall, the duration of the experiment in our model is consistent with the timing of events that take place in an urban setting with a trauma victim who sustains a penetrating torso injury. Enough time was allotted to simulate emergency room exams, intubation, anesthesia, operating room set-up, incision, and vascular control of a bleeding site $(14,15)$. The model, however, was not intended to simulate the timing of events of an emergency room thoracotomy, a scenario of a patient in extremis.

Our model causes a severe hemorrhagic shock as shown by the significant decrease in MAP, hemoglobin and hematocrit, by abnormal base excess, and by a $20 \%$ mortality rate within the first $15 \mathrm{~min}$. The average intra-abdominal blood loss of $30 \mathrm{~mL}$ represents approximately $20 \%$ of the total blood volume of the animal, and corresponds to a class II shock as per the Advanced Trauma Life Support protocol of the American College of Surgeons (13). With the addition of tissue injury provoked by laparotomy, a severe shock is achieved in the model, to the extent that, in a pilot study previously performed by us, there was $100 \%$ mortality within 30 min after aortic injury if no resuscitation fluid at all was given to the animals, underscoring the importance of the infusion of at least some fluid during the treatment of severe hemorrhagic shock.

Other experimental models involving tail amputation or hemorrhage by controlled blood withdrawal do not reproduce faithfully the events of major penetrating torso trauma and the resulting uncontrolled hemorrhage. Additionally, such models produce only minimal tissue injury $(11,19-21)$. In our model, however, significant tissue injury was created by both the invasive pre-shock surgical procedures and, more importantly, by the laparotomy. The latter was closed swiftly, immediately after the aortic injury to mimic the tamponade effect of the abdominal wall and provided an adequate environment for clot formation in abdominal injury.

Acute fluid management of the bleeding trauma patient plays a major role in the hemostatic response. Therefore, a clinically relevant animal model of hemorrhagic shock should be in keeping with the current resuscitation practice. The primary resuscitation strategy of severe hemorrhage is to administer large volumes of crystalloids and packed red blood cells. In our model, fluid resuscitation reproduced the average crystalloid infusion by EMS during transport, approximately $1.7 \mathrm{~mL} \cdot \mathrm{kg}^{-1} \cdot \mathrm{min}^{-1}$ or a maximum of $5 \mathrm{~mL} / \mathrm{min}$ of LR infusion in the case of a $2.5-\mathrm{kg}$ rabbit $(14,15,22)$.

Another resuscitation regimen considered in our model is $\mathrm{HR}$, a strategy known since World War I but only recently applied to the clinical management of trauma patients with penetrating torso injuries $(3,13,23-25)$. Previous experimental models of hemorrhagic shock have demonstrated that HR leads to less blood loss and ultimately lower mortality when compared to NR (8), although in the present study there were no deaths among animals receiving either resuscitation regimen. 
HR addresses at least two important factors that affect the hemostatic response: reduces dilution of pro-coagulants and dislodgment of the clot from the site of injury $(3,8,13,23-$ 25). A study using a computer-based model of uncontrolled hemorrhagic shock and resuscitation concluded that a rapidly bleeding injured patient bleeds for approximately $5.5 \mathrm{~min}$ before intrinsic hemostasis is achieved (15). Thus, by the time EMS arrives at the scene, in most cases bleeding decreased significantly and "self-resuscitation" has already begun (15). However, the recently formed clot is fragile and vulnerable to blood pressure changes caused by overzealous fluid resuscitation. Thus, an increase in blood pressure can easily dislodge the clot from the vessel causing rebleeding, a phenomenon known as the "leaky bucket" theory (15).

As mentioned previously, the present data support the concept of ongoing self-resuscitation, especially noticed between five and 15 min after injury to the aorta. Furthermore, our data also support the fact that rebleeding increased after the 50th min in normotensive resuscitated animals, and intra-abdominal blood loss was significantly greater compared to HR animals. Interestingly, another study comparing no fluid, small volume infusion of hypertonic saline dextran $(7.5 \% \mathrm{NaCl} / 6 \%$ dextran 70$)$, and a large volume of LR solution demonstrated no difference in blood loss (19). In that study, however, the intra-abdominal blood loss was assessed after only 20 min from the start of the resuscitation phase and may not have allotted enough time to detect rebleeding, and consequently a difference in blood loss between groups (19).

Even though it was not the purpose of this study to investigate the coagulopathy related to hemorrhagic shock, we believe that the model described herein sets the stage for such an investigation $(4,9)$. Using basic routine laboratory coagulation assays, we demonstrated that hemorrhagic shock is associated with a significant decrease in platelet count independent of the resuscitation strategy used. We speculate that platelets were less dysfunctional among animals undergoing permissive hypotension resuscitation, arguably contributing to the reduced intra-abdominal blood loss when compared to the NR group. The low levels of antithrombin also suggest the role of dilutional coagulopathy particularly among the NR group animals. The fact that the aPTT remained within normal limits in both groups coincides with recent clinical observations demonstrating that this test

\section{References}

1. Krug EG, Sharma GK, Lozano R. The global burden of injuries. Am J Public Health 2000; 90: 523-526.

2. Hoyt DB, Bulger EM, Knudson MM, Morris J, lerardi R, Sugerman $\mathrm{HJ}$, et al. Death in the operating room: an analysis of a multi-center experience. J Trauma 1994; 37: 426-432.

3. Bickell WH, Wall MJ Jr, Pepe PE, Martin RR, Ginger VF, AlIen MK, et al. Immediate versus delayed fluid resuscitation is a poor indicator of the hemostatic disturbances that take place in traumatic hemorrhage $(26,27)$.

Bleeding is among the easiest forms of insults that can be reproduced in experimental models and has been studied for more than 80 years. However, all animal models, including ours, have important limitations, the need for anesthesia being one of them. Although the cardiovascular effect of xylazine has been shown to be negligible in conscious large animal experiments, ketamine undoubtedly compromises mean arterial pressure, oxygen consumption, as well as cardiac and pulmonary performance $(28,29)$. Therefore, the pattern of response to hemorrhagic shock with either hypotensive or normotensive resuscitation could have been different if no anesthesia had been used.

Although the present model has the advantage of using a small animal such as the rabbit, which is more cost-effective and easier to manage, larger animals have cardiovascular and hemodynamic responses to shock more similar to those of humans. Nonetheless, a recent report concluded that the hemostatic response of the rabbit is physiologically one of the most similar to the human response (30).

Our study has another limitation related to most animal models, which is the difficulty to reproduce the late complications of shock and resuscitation of trauma patients (21). The total time period of this experiment $(85 \mathrm{~min}$ ) enabled us to study only the acute phase of hemorrhagic shock, which represents a small window in a very complex chain of events. Finally, even though a model of uncontrolled bleeding is considered to be more clinically relevant than controlled hemorrhage models, it tends to produce inconsistent results with respect to the extent of bleeding, reduction of blood pressure, and survival (31).

Our rabbit model reproduces the sequence of events from pre-hospital to early in-hospital care of penetrating trauma and uncontrolled intra-abdominal hemorrhage caused by an arterial injury in a clinically relevant fashion. Using this model, we were able to demonstrate that hypotensive resuscitation results in less intra-abdominal blood loss than normotensive resuscitation, possibly by preventing re-bleeding episodes.

\section{Acknowledgments}

Research supported by CAPES (\#0017/08-6) and CNPq

for hypotensive patients with penetrating torso injuries. $N$ Engl J Med 1994; 331: 1105-1109.

4. Hoyt DB, Dutton RP, Hauser CJ, Hess JR, Holcomb JB, Kluger $Y$, et al. Management of coagulopathy in the patients with multiple injuries: results from an international survey of clinical practice. J Trauma 2008; 65: 755-764.

5. Moore FA, McKinley BA, Moore EE. The next generation in 
shock resuscitation. Lancet 2004; 363: 1988-1996.

6. Cho SD, Holcomb JB, Tieu BH, Englehart MS, Morris MS, Karahan ZA, et al. Reproducibility of an animal model simulating complex combat-related injury in a multiple-institution format. Shock 2009; 31: 87-96.

7. Majde JA. Animal models for hemorrhage and resuscitation research. J Trauma 2003; 54: S100-S105.

8. Mapstone J, Roberts I, Evans P. Fluid resuscitation strategies: a systematic review of animal trials. J Trauma 2003; 55: 571-589.

9. Parr MJ, Bouillon B, Brohi K, Dutton RP, Hauser CJ, Hess JR, et al. Traumatic coagulopathy: where are the good experimental models? J Trauma 2008; 65: 766-771.

10. Tsukamoto T, Pape HC. Animal models for trauma research: what are the options? Shock 2009; 31: 3-10.

11. Capone A, Safar P, Stezoski SW, Peitzman A, Tisherman $S$. Uncontrolled hemorrhagic shock outcome model in rats. Resuscitation 1995; 29: 143-152.

12. Sondeen JL, Dubick MA, Holcomb JB, Wade CE. Uncontrolled hemorrhage differs from volume- or pressurematched controlled hemorrhage in swine. Shock 2007; 28: 426-433.

13. Committee on Trauma. Shock. In: Anonymous, Advanced Trauma Life Support Student Manual. 8th edn. Chicago: American College of Surgeons; 2008. p 55-84.

14. Cayten CG, Longmore W, Kuehl A. BLS versus ALS for trauma in an urban setting. Ann Emerg Med 1986; 15: 626627.

15. Hirshberg A, Hoyt DB, Mattox KL. Timing of fluid resuscitation shapes the hemodynamic response to uncontrolled hemorrhage: analysis using dynamic modeling. J Trauma 2006; 60: 1221-1227.

16. Shaftan GW, Chiu CJ, Dennis C, Harris B. Fundamentals of physiologic control of arterial hemorrhage. Surgery 1965; 58: 851-856.

17. Henderson KI, Coats TJ, Hassan TB, Brohi K. Audit of time to emergency trauma laparotomy. Br J Surg 2000; 87: 472476.

18. Snyder CW, Weinberg JA, McGwin G Jr, Melton SM, George $\mathrm{RL}$, Reiff $\mathrm{DA}$, et al. The relationship of blood product ratio to mortality: survival benefit or survival bias? J Trauma 2009; 66: 358-362.

19. Bruscagin V, de Figueiredo LF, Rasslan S, Varicoda EY, Rocha e Silva M. Fluid resuscitation improves hemodynamics without increased bleeding in a model of uncontrolled hemorrhage induced by an iliac artery tear in dogs. J Trauma
2002; 52: 1147-1152.

20. Fabian TC, Croce MA, Stewart RM, Dockter ME, Proctor KG. Neutrophil CD18 expression and blockade after traumatic shock and endotoxin challenge. Ann Surg 1994; 220: 552561.

21. Rezende-Neto JB, Moore EE, Masuno T, Moore PK, Johnson JL, Sheppard FR, et al. The abdominal compartment syndrome as a second insult during systemic neutrophil priming provokes multiple organ injury. Shock 2003; 20: 303-308.

22. Soucy DM, Rude M, Hsia WC, Hagedorn FN, Illner H, Shires GT. The effects of varying fluid volume and rate of resuscitation during uncontrolled hemorrhage. J Trauma 1999; 46: 209-215.

23. Canon WB, Fraser J, Cowell EM. The preventive treatment of wound shock. JAMA 1918; 618-621.

24. Holcomb JB, Jenkins D, Rhee P, Johannigman J, Mahoney $P$, Mehta $S$, et al. Damage control resuscitation: directly addressing the early coagulopathy of trauma. J Trauma 2007; 62: 307-310.

25. Tremblay LN, Rizoli SB, Brenneman FD. Advances in fluid resuscitation of hemorrhagic shock. Can J Surg 2001; 44: 172-179.

26. Martini WZ, Cortez DS, Dubick MA, Park MS, Holcomb JB. Thrombelastography is better than PT, aPTT, and activated clotting time in detecting clinically relevant clotting abnormalities after hypothermia, hemorrhagic shock and resuscitation in pigs. J Trauma 2008; 65: 535-543.

27. Plotkin AJ, Wade CE, Jenkins DH, Smith KA, Noe JC, Park $\mathrm{MS}$, et al. A reduction in clot formation rate and strength assessed by thrombelastography is indicative of transfusion requirements in patients with penetrating injuries. $J$ Trauma 2008; 64: S64-S68.

28. Grant C, Upton RN. Cardiovascular and haemodynamic effects of intramuscular doses of xylazine in conscious sheep. Aust Vet J 2001; 79: 58-60.

29. Waxman K, Shoemaker WC, Lippmann M. Cardiovascular effects of anesthetic induction with ketamine. Anesth Analg 1980; 59: 355-358.

30. Siller-Matula JM, Plasenzotti R, Spiel A, Quehenberger $P$, Jilma B. Interspecies differences in coagulation profile. Thromb Haemost 2008; 100: 397-404.

31. Bickell WH, Bruttig SP, Wade CE. Hemodynamic response to abdominal aortotomy in the anesthetized swine. Circ Shock 1989; 28: 321-332. 